EPJ Web of Conferences will be set by the publisher

DOI: will be set by the publisher

(C) Owned by the authors, published by EDP Sciences, 2014

\title{
Axial magnetic effect in two-color quenched lattice QCD
}

\author{
V. Braguta ${ }^{1,2}$, M. N. Chernodub ${ }^{3,4,5}$, V. A. Goy ${ }^{6}$, K. Landsteiner ${ }^{6}$, A. V. Molochkov ${ }^{5}$, and M. I. \\ Polikarpov ${ }^{1,7}$ \\ ${ }^{1}$ IHEP, Protvino, Moscow region, 142284 Russia \\ ${ }^{2}$ ITEP, B. Cheremushkinskaya street 25, Moscow, 117218 Russia \\ ${ }^{3}$ CNRS, Laboratoire de Mathématiques et Physique Théorique, Université \\ François-Rabelais Tours, Fédération Denis Poisson, Parc de Grandmont, 37200 Tours, France \\ ${ }^{4}$ Department of Physics and Astronomy, University of Gent, Krijgslaan 281, S9, B-9000 Gent, Belgium \\ ${ }^{5}$ School of Biomedicine, Far Eastern Federal University, Sukhanova str., 8, Vladivostok, 690950, Russia \\ ${ }^{6}$ School of Natural Sciences, Far Eastern Federal University, Sukhanova str., 8, Vladivostok, 690950, Russia \\ ${ }^{7}$ Instituto de Física Teórica UAM/CSIC, C/ Nicolás Cabrera 13-15, Universidad Autónoma de Madrid, Can- \\ toblanco, 28049 Madrid, Spain \\ ${ }^{8}$ Moscow Inst of Physics and Technology, Institutskii per. 9, Dolgoprudny, Moscow Region, 141700, Russia
}

\begin{abstract}
The Axial Magnetic Effect manifests itself as an equilibrium energy flow of massless fermions induced by the axial (chiral) magnetic field. Here we study the Axial Magnetic Effect in the quenched SU(2) lattice gauge theory with massless overlap fermions at finite temperature. We numerically observe that in the low-temperature hadron phase the effect is absent due to the quark confinement. In the high-temperature deconfinement phase the energy flow is an increasing function of the temperature which reaches the predicted asymptotic $T^{2}$ behavior at high temperatures. We find, however, that energy flow is about one order of magnitude lower compared to a theoretical prediction.
\end{abstract}

\section{Introduction}

A standard manifestation of an anomaly in a quantum field theory is a breaking of a classical symmetry by quantum fluctuations. As a consequence, a classically conserved current, associated with anomalous symmetry, is no more conserved on a quantum level. For example, the axial anomaly leads to a non-conservation of the axial current (difference in current of left- and right-handed massless fermions). A physically measurable consequence of the axial anomaly is the decay of the neutral pion into two photons.

Recently it was realized that the quantum anomalies are also important for certain unusual transport effects characterized by the existence of dissipationless currents in the presence external fields. It is very interesting that these nondissipative currents exist in (strongly) interacting systems in thermal equilibrium. The celebrated examples of the anomalous transport properties are the Chiral Magnetic Effect [1-6] and the Chiral Vortical Effect [7-11]. The dissipationless currents can either be anomalous currents associated with global symmetries like the axial current or the currents related to the presence of gauged symmetries such as the electric current or the energy current. 
The anomalous transport phenomena can be described by a set of transport coefficients which relate the dissipationless currents with external fields. It is interesting to notice that the dependence of the transport coefficients on chemical potentials is associated with conventional chiral anomalies while a temperature dependence enters via a gravitational contribution to the anomalies [12-14].

For example, the axial anomaly leads to the Chiral Magnetic Effect (CME): in the background of an external magnetic field a chirally imbalanced gas of fermions generates an electric current along the axis of the magnetic field according to the anomalous conduction law

$$
\vec{j}=\sigma_{A M E} \vec{B}
$$

The chiral imbalance, given by the difference in densities of left- and right-handed fermions, is usually described by the presence of a nonzero chiral chemical potential $\mu_{5}$ and $\sigma_{A M E} \propto \mu_{5}$.

The CME was observed in numerical simulations of lattice QCD $[15,16]$. The aim of our study is to demonstrate, in thermal equilibrium, the existence of the gravitationally induced anomalous dissipationless transport using first-principle lattice simulations.

The gravitational anomaly can be probed by the Axial Magnetic Effect which generates the energy flow of massless fermions along the direction of the axial magnetic field $\vec{B}_{5}$. The axial magnetic field is a magnetic field which distinguishes the chiralities of fermions. This field acts as $+\vec{B}_{5}\left(-\vec{B}_{5}\right)$ on a left- (right-)handed fermion.

The Axial Magnetic Effect is convenient for investigation in lattice gauge theories because the corresponding anomalous transport coefficient is nonzero even in the absence of chemical potentials. In other words, in order to probe this effect in lattice simulations it is sufficient to consider a finite temperature lattice gauge theory with all chemical potentials set to zero. The axial magnetic field can trivially be introduced as the usual magnetic field acting separately on left- and right-handed fermions with opposite signs. This strategy was implemented in Refs. [17, 18] which will be summarized below.

\section{Axial Magnetic Effect in QCD}

The AME is described by the following equation:

$$
\vec{J}_{\epsilon}=\sigma_{\mathrm{AME}} \vec{B}_{5},
$$

where $J_{\epsilon}^{i}=T^{0 i}$ is the energy flow, $\vec{B}_{5}$ is the axial magnetic field. The AME may be realized in the pure thermal vacuum with all chemical potentials set to zero, $\mu=\mu_{5}=0$. In a weakly coupled gas of left- and right-handed fermions with charges, respectively, $q_{L}$ and $q_{R}$, the axial magnetic conductivity raises with temperature as follows:

$$
\sigma_{\mathrm{AME}}=\frac{1}{24}\left(\sum_{R} q_{R}-\sum_{L} q_{L}\right) T^{2} .
$$

It is relatively simple to check the AME transport law (2) in Euclidean lattice simulations because the lattice implementation of the axial magnetic field $\vec{B}_{5}$ is a rather straightforward procedure [17-20]. This fact was used in Ref. [19, 20] where the validity of the theoretical prediction (3) was explicitly demonstrated in numerical simulations of a system of free lattice fermions. In a case of (strongly) interacting fermions the law (3) may in principle be modified. The central issue of the present article is a numerical calculation of the axial magnetic conductivity entering the transport law (2) in a strongly interacting theory. 
We study the AME in a simplest possible nonperturbative setup given by a two-color QCD with quenched quarks. The word "quenched" means that quark loops are ignored so that the effect of the virtual quarks on gluon dynamics is neglected. It is known that the quark propagator is not strongly altered by the quenching effects [21] therefore one may generally expect that the quenching should give a moderate contribution to the anomalous transport of quarks in Eq. (6).

The quenched SU(2) QCD has two phases, the low-temperature confinement (hadron) phase and the high-temperature deconfinement (plasma) phase. The phases are separated by a second-order phase transition at the critical temperature $T=T_{c}$.

On general grounds one can expect that in the confinement phase $T<T_{c}$ the dissipationless energy current should not be generated since in the confinement phase the free massless quarks - which are critically important for the AME - cannot exist as asymptotic states.

The AME may only be realized in the deconfinement phase at $T>T_{c}$. Generally, on dimensional arguments, one may expect that in the high-temperature regime at $T \gg T_{c}$ the AME conductivity should be proportional to the squared temperature $\sigma_{\mathrm{AME}} \sim T^{2}$ as in the theoretical formula (3).

In order to illustrate the existence of the effect it is enough to consider one fermion flavor, $N_{f}=1$, which carries a unit charge:

$$
q_{5}^{L}=-q_{5}^{R}=+e .
$$

According to Eq. (3), in QCD with two colors $N_{c}=2$ and one flavor $N_{f}=1$ the prefactor $\sigma$ in the AME law (2) has the following form:

$$
\sigma^{\text {th }}(T)=C_{\mathrm{AME}}^{\text {th }} T^{2},
$$

with

$$
C_{\mathrm{AME}}^{\mathrm{th}}=\frac{2 N_{f} N_{c}}{24} \equiv \frac{1}{6} .
$$

\section{Numerical results}

We consider the following Lagrangian,

$$
\mathcal{L}_{5}=\bar{\psi}\left(\partial_{\mu}-i g A_{\mu}^{a} t^{a}-i \gamma^{5} e A_{5, \mu}\right) \gamma^{\mu} \psi \equiv \bar{\psi} \mathscr{D}_{5}\left(A_{5}\right) \psi,
$$

where the axial field $A_{5, \mu}$ acts as a classical Abelian background field superimposed over the dynamical non-Abelian gauge field $A_{\mu}^{a}$, and $t^{a}, a=1,2,3$ are the generators of the corresponding SU(2) gauge group. The gauge field $A_{\mu}^{a}$ is generated in standard lattice Monte-Carlo simulations of SU(2) lattice gauge theory.

We choose the axial gauge field in the following form

$$
A_{5,0}=A_{5,3}=0, \quad A_{5,1}=-\frac{x_{2} B_{5}}{2}, \quad A_{5,2}=\frac{x_{1} B_{5}}{2},
$$

which corresponds to a stationary uniform axial magnetic field pointing in the third direction, $B_{5, i}=$ $B_{5} \cdot \delta_{i, 3}$. The latin index $i=1,2,3$ labels the spatial coordinates and $\mu=0$ is the time direction.

The energy flow of the quarks is given by the vacuum expectation value of the $T^{0 i}$ component of the stress-energy tensor,

$$
J_{\epsilon}^{i}=\left\langle T^{0 i}\right\rangle \equiv \frac{i}{2}\left\langle\bar{\psi}\left(\gamma^{0} \mathcal{D}_{5}^{i}+\gamma^{i} \mathcal{D}_{5}^{0}\right) \psi\right\rangle,
$$

where the covariant derivative is defined in Eq. (7). 
In addition to the fermionic part (9), the energy flow may generally also contain a gluonic contribution. However, in the quenched approach the quark vacuum loops are absent so that the gluons are not sensitive to the external magnetic field (although the gluons carry no electric charge, their dynamics is affected by the external magnetic field via interactions with quark vacuum loops). As a result the energy flow of gluons is vanishing in our approach and Eq. (9) gives us the full energy flow in the theory.

The details of the numerical calculation of the energy flow (9) in the theory (7) in the presence of the axial magnetic field given by the background (8) can be found in Refs. $[17,18]$. Below we summarize the basic results of our studies.

First of all, we have numerically observed in Ref. [17] that in the confinement phase the AME is absent, as expected. In the deconfinement phase the dissipationless energy flow is a linear function of the strength of the axial magnetic field $B_{5}$. This linear behavior is valid a wide set of temperature and volumes, in agreement with the theoretical prediction (2). A corresponding example is shown in Fig. 1 for the fixed temperature $T \approx 1.6 T_{c}$.

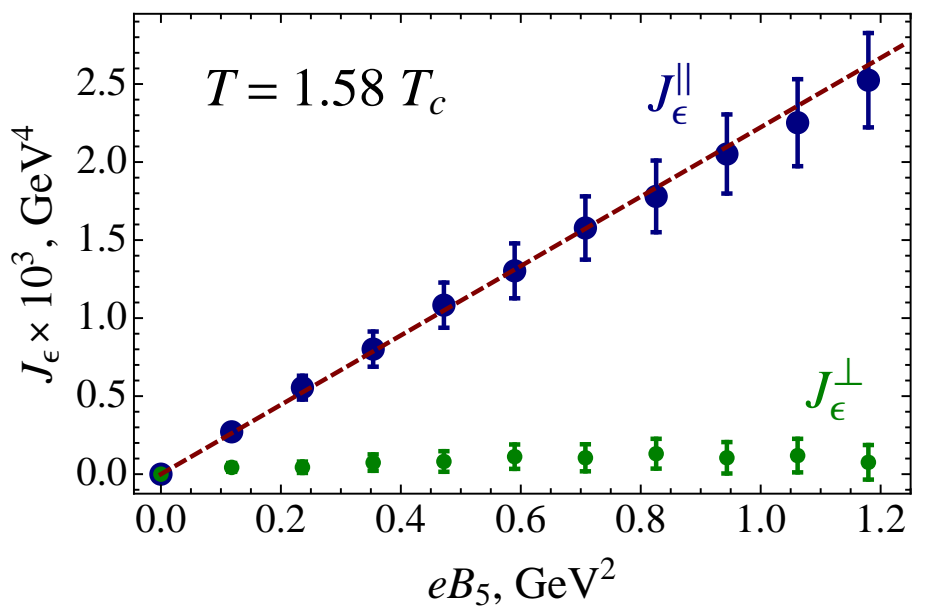

Figure 1. Energy flow (9) parallel $\left(J_{\epsilon}^{\|}\right)$and perpendicular $\left(J_{\epsilon}^{\perp}\right)$ to the direction of the axial magnetic field $B_{5}$ in the deconfinement phase at $T \approx 1.6 T_{c}$. The red dashed line represents the best linear fit confirming the existence of the AME (2). From Ref. [17].

Due to the robustness of the AME's linear law in order to find the temperature behavior of the conductivity coefficient,

$$
C_{\mathrm{AME}}(T)=\frac{J_{\epsilon}\left(T, e B_{5}\right)}{e B_{5} T^{2}},
$$

it is sufficient to calculate the energy current $J_{\epsilon}$ for a single value of the external axial magnetic field $B_{5}$ at a given temperature $T$. In Fig. 2 we show the dimensionless coefficient (10) of the conductivity (3) as a function of temperature $T$. It is seen that the conductivity coefficient $C_{\mathrm{AME}}(T)$ raises with temperature at phase transition region, and approaches a constant value at $T \sim 1.5 T_{c}$ implying the expected $T^{2}$ behavior of the conductivity $\sigma(T)$ at higher temperatures [18]. 


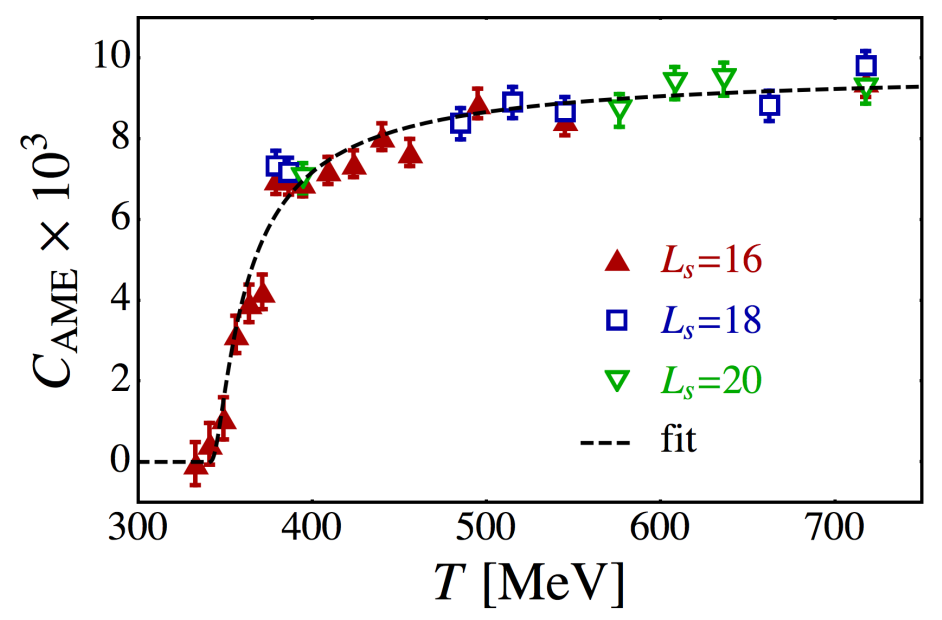

Figure 2. The dimensionless conductivity coefficient (10) of the dissipationless energy flow vs. temperature. The dashed line represents the best fit by Eq. (11). From Ref. [18].

It turns out that the temperature behavior of the coefficient $C_{\mathrm{AME}}$ can well be described by the following formula [18]

$$
C_{\mathrm{AME}}^{\mathrm{fit}}(T)=C_{\mathrm{AME}}^{\infty} \exp \left(-\frac{h T_{0}}{T-T_{0}}\right),
$$

valid for $T>T_{0}$. The corresponding best fit is shown in Fig. 1 by the dashed line. It is important to stress that the best fit parameter

$$
C_{\mathrm{AME}}^{\infty}=0.0097(2),
$$

corresponds to the AME conductivity

$$
\sigma(T)=C_{\mathrm{AME}}^{\infty} T^{2}
$$

in the high-temperature limit. The numerical value of this quantity is much smaller than the theoretical prediction (6). Indeed, the theoretical proportionality coefficient should be an order of magnitude larger (6):

$$
C_{\mathrm{AME}}^{\mathrm{th}}=\frac{1}{6} \approx 0.166 .
$$

We attribute this difference to effects of nonperturbative interactions because in lattice simulations of free (noninteracting) fermions the anomalous energy flow agrees very well with the theoretical prediction [19].

\section{Conclusions}

Summarizing, we have numerically studied the Axial Magnetic Effect in the quenched lattice SU(2) gauge theory. The energy flow is absent in the confinement phase while in the deconfinement phase the conductivity flow is proportional to the strength of the axial magnetic field, as expected. The 
AME conductivity raises sharply in the phase transition region at $T \sim T_{c}$ and reaches the expected asymptotic $T^{2}$ behavior at high temperatures. However, the conductivity coefficient found in our numerical simulations is approximately 17 times smaller than the coefficient predicted by the linear response theory at weak coupling.

\section{Acknowledgements}

The Moscow group was supported by Grants RFBR-13-02-01387, RFBR-14-02-01185, MD3215.2014.2, Federal Special-Purpose Program "Cadres" of the Russian Ministry of Science and Education, and by a grant from the FAIR-Russia Research Center. Numerical calculations were performed at the ITEP computer systems "Graphyn" and "Stakan". The work of M.N.C. was partially supported by grant ANR-10-JCJC-0408 HYPERMAG. The work of K.L. was partially supported by Plan Nacional de Altas Energí FPA2012-32828, Consolider Ingenio 2010 CPAN (CSD2007-00042); HEP-HACOS S2009/ESP-1473, SEV-2012-0249. This project was also supported by the Far Eastern Federal University, grant No. 13-09-617-m_a.

\section{References}

[1] K. Fukushima, D. E. Kharzeev and H. J. Warringa, Phys. Rev. D 78, 074033 (2008).

[2] D. E. Kharzeev, L. D. McLerran and H. J. Warringa, Nucl. Phys. A 803, 227 (2008).

[3] A. Vilenkin, Phys. Rev. D 22, 3080 (1980).

[4] M. A. Metlitski and A. R. Zhitnitsky, Phys. Rev. D 72, 045011 (2005).

[5] M. Giovannini and M. E. Shaposhnikov, Phys. Rev. D 57, 2186 (1998).

[6] A. Y. Alekseev, V. V. Cheianov and J. Frohlich, Phys. Rev. Lett. 81, 3503 (1998).

[7] J. Erdmenger, M. Haack, M. Kaminski and A. Yarom, JHEP 0901 (2009) 055.

[8] N. Banerjee, J. Bhattacharya, S. Bhattacharyya, S. Dutta, R. Loganayagam and P. Surowka, JHEP 1101 (2011) 094.

[9] D. T. Son and A. R. Zhitnitsky, Phys. Rev. D 70, 074018 (2004).

[10] A. Vilenkin, Phys. Rev. D 20 (1979) 1807.

[11] A. Vilenkin, Phys. Rev. D 21 (1980) 2260.

[12] K. Landsteiner, E. Megias and F. Pena-Benitez, Phys. Rev. Lett. 107, 021601 (2011).

[13] K. Landsteiner, E. Megias and F. Pena-Benitez, Lect. Notes Phys. 871 (2013) 433.

[14] K. Landsteiner, E. Megias, L. Melgar and F. Pena-Benitez, JHEP 1109 (2011) 121.

[15] P. V. Buividovich, M. N. Chernodub, E. V. Luschevskaya and M. I. Polikarpov, Phys. Rev. D 80, 054503 (2009).

[16] A. Yamamoto, Phys. Rev. Lett. 107, 031601 (2011).

[17] V. Braguta, M. N. Chernodub, K. Landsteiner, M. I. Polikarpov and M. V. Ulybyshev, Phys. Rev. D 88, 071501 (2013).

[18] V. Braguta, M. N. Chernodub, V. A. Goy, K. Landsteiner, A. V. Molochkov and M. I. Polikarpov, Phys. Rev. D 89, 074510 (2014).

[19] P. V. Buividovich, Nucl. Phys. A 925, 218 (2014);

[20] P. V. Buividovich, arXiv:1309.4966 [hep-lat].

[21] P. O. Bowman, U. M. Heller, D. B. Leinweber, M. B. Parappilly, A. G. Williams, J. Zhang, Phys. Rev. D 71, 054507 (2005). 